\title{
On the local property of the absolute summability $|C, \alpha|$ for Fourier series.
}

\author{
By Mineo KIYOHARA ${ }^{1)}$
}

(Received Oct. 1, 1957)

1. V. A. Magarik [4] has generalized Wiener's theorem ${ }^{2}$ on the absolute convergence of Fourier series to the absolute summability $|C, \alpha|$. His assertion is as follows:

Let $f(x)$ be Lebesgue integrable in the interval $(-\pi, \pi)$ and periodic with period $2 \pi$. If at every point $y$ on the closed interval $[-\pi, \pi]$ there are a function $g_{y}(x)$ and $a \delta>0$ such that (i) $g_{y}(x)=f(x)$ for $|x-y|<\delta$, and (ii) both the Fourier series of $g_{y}(x)$ and its conjugate series are absolutely summable $|C, \alpha|,{ }^{3)}$ then the Fourier series of $f(x)$ is absolutely summable $|C, \alpha|$, where $\alpha \geqq 0$.

For the case $\alpha=1$, W. C. Randels [5] proved this proposition without the condition on the absolute summability $|C, 1|$ for the conjugate series.

In the present note, we shall show that the condition on the absolute summability for the conjugate series is also superfluous for the general case; that is, the following theorem will be established.

Theorem. Let $f(x)$ be Lebesgue integrable in the interval $(-\pi, \pi)$ and periodic with period $2 \pi$. If at every point $y$ on the closed interval $[-\pi, \pi]$ there are a function $g_{y}(x)$ and $a \delta>0$ such that (i) $g_{y}(x)=f(x)$ for $|x-y|<\delta$ and (ii) the Fourier series of $g_{y}(x)$ is absolutely summable $|C, \alpha|$, then the Fourier series of $f(x)$ is absolutely summable $|C, \alpha|$, where $\alpha \geqq 0$.

2. The case for $\alpha>1$ of our theorem follows immediately from the known theorem of L.S. Bosanquet [1]:

The absolute summability $|C, \alpha|, \alpha>1$, for Fourier series of a Lebesgue integrable function with period $2 \pi$ at a point $x=x_{0}$ depends only on the behaviour of the generating function in the neighbourhood of the point $x_{0}$.

On the other hand, L.S. Bosanquet and H. Kestelman [2] proved that the mentioned result of L.S. Bosanquet does not hold for $\alpha=1$.

Thus, it is the case $0 \leqq \alpha \leqq 1$ in which we are interested. However, it

1) The author wishes to thank Dr. S. Yano for his valuable advice during the preparation of this paper.

2) A. Zygmund [6], p. 140.

3) For the definition of absolute summability $|C, \alpha|$, see below. 
will be sufficient to prove our theorem only for $0<\alpha<1$, because the cases for $\alpha=0$ and $\alpha=1$ were already proved by N. Wiener and W. C. Randels respectively.

We must now make some general remarks about the absolute summability $|C, \alpha|$.

Let $\alpha$ be any real number and put

$$
A_{n}^{\alpha}=\left(\begin{array}{c}
\alpha+n \\
n
\end{array}\right)=\frac{(\alpha+1)(\alpha+2) \cdots(\alpha+n)}{n !} \quad(n>0)
$$

(1)

$$
A_{0}^{\infty}=1 \text {. }
$$

Then, it is well known ${ }^{4}$ that if $\alpha \neq-1,-2, \cdots$

$$
A_{n}^{\alpha} \cong \frac{n^{\alpha}}{\Gamma(\alpha+1)}
$$

For any given series $\sum_{n=0}^{\infty} x_{n}$ and any $n \geqq 0$ we write

$$
\sigma_{n}^{\alpha}=\frac{1}{A_{n}^{\alpha}} \sum_{k=0}^{n} A_{n-k}^{\alpha} x_{k} .
$$

The series $\sum_{n=0}^{\infty} x_{n}$ is said to be absolutely summable $|C, \alpha|$, if the series

$$
\sum_{n=1}^{\infty}\left|\sigma_{n}^{\alpha}-\sigma_{n-1}^{\alpha}\right|
$$

is convergent.

We shall need the next lemmas which are due to M. E. Kogbetliantz [3].

Lemma 1. The series $\sum_{n=0}^{\infty} x_{n}$ is absolutely summable $|C, \alpha|$ if and only if the series

$$
\sum_{n=0}^{\infty} \frac{1}{A_{n}^{\alpha+1}}\left|\sum_{k=0}^{n} k A_{n-k}^{\alpha-1} x_{k}\right|
$$

is convergent, where $\alpha \neq-1,-2, \cdots$.

Lемма 2. If the series $\sum_{n=0}^{\infty} x_{n}$ is absolutely summable $|C, \alpha|$, then the series (6)

$$
\sum_{n=0}^{\infty} \frac{\left|x_{n}\right|}{(n+1)^{\infty}}
$$

is convergent, where $\alpha \geqq 0$.

In order to apply this definition to Fourier series in the exponential form we put

4) A. Zygmund [6], p. 42 . 


$$
x_{n}=\left(c_{n} e^{2 n x}+c_{-n} e^{-i n x}\right) .
$$

From Lemma 2 it follows that if a Fourier series is absolutely summable $|C, \alpha|$ over any interval $(a, b)$, then the series

$$
\sum_{n=-\infty}^{\infty} \frac{\left|c_{n}\right|}{(|n|+1)^{\alpha}}
$$

is convergent, where $c_{n}$ 's are the Fourier coefficients and $\alpha \geqq 0$.

3. Let us proceed to the proof of Theorem. We may suppose $0<\alpha<1$ as it was remarked in the above section.

By the Heine-Borel covering theorem and the hypothesis of our theorem there exist a finite number of overlapping intervals $\left(\delta_{i}, \delta_{i}{ }^{\prime}\right)$ covering $(-\pi, \pi)$ and functions $g_{i}(x)$ such that the Fourier series of $g_{2}(x)$ is absolutely summable $|C, \alpha|$ and $g_{i}(x)=f(x)$ on $\left(\delta_{i}, \delta_{i}{ }^{\prime}\right)$. These intervals may be chosen so that $\delta_{\imath}<\delta_{i-1}{ }^{\prime}<\delta_{i+1}<\delta_{i}{ }^{\prime}$.

The functions $h_{2}(x)$ are now defined by

$$
h_{i}(x)= \begin{cases}A_{\imath}\left(x-\delta_{\imath}\right)^{3}+B_{\imath}\left(x-\delta_{\imath}\right)^{2}, & \delta_{i} \leqq x<\delta_{\imath-1}{ }^{\prime}, \\ 1, & \delta_{i-1} \leqq x<\delta_{\imath+1}, \\ 1-h_{i+1}(x), & \delta_{i+1} \leqq x<\delta_{\imath}{ }^{\prime}, \\ 0, & x<\delta_{i} \text { or } \delta_{i}{ }^{\prime} \leqq x, \\ h_{\imath}(x+2 \pi), & \end{cases}
$$

where $A_{i}, B_{\imath}$ are defined by the relations

$$
\begin{aligned}
& 3 A_{i}\left(\delta_{\imath-1}{ }^{\prime}-\delta_{\imath}\right)+2 B_{\imath}=0, \\
& A_{\imath}\left(\delta_{i-1}{ }^{\prime}-\delta_{\imath}\right)^{3}+B_{\imath}\left(\delta_{i-1}{ }^{\prime}-\delta_{i}\right)^{2}=1 .
\end{aligned}
$$

The second relation of (9) implies that $h_{i}(x)$ is continuous, and by the first relation we see that

$$
h_{\imath}{ }^{\prime}\left(\delta_{\imath}\right)=h_{i}{ }^{\prime}\left(\delta_{i-1}{ }^{\prime}\right)=h_{\imath}{ }^{\prime}\left(\delta_{i+1}\right)=h_{\imath}{ }^{\prime}\left(\delta_{i}\right)=0,
$$

so that $h_{i}^{\prime}(x)$ is absolutely continuous and $h^{\prime \prime}(x)$ is of bounded variation. Therefore the Fourier coefficients of $h_{\imath}(x)$ are

$$
\begin{aligned}
c_{n}\left(h_{i}\right) & =\frac{1}{2 \pi} \int_{-\pi}^{\pi} h_{2}(x) e^{-i n x} d x \\
& =-\frac{1}{2 \pi} \int_{-\pi}^{\pi} \frac{h_{i}{ }^{\prime \prime}(x)}{n^{2}} e^{-i n x} d x \\
& =O\left(n^{-3}\right) .
\end{aligned}
$$

It is also clear that

$$
\sum h_{i}(x)=1
$$


and

$$
f(x)=\sum_{i} g_{i}(x) h_{i}(x) .
$$

The Fourier coefficients of $g_{i}(x) h_{i}(x)$ will be given by

$$
c_{n}\left(g_{i} \cdot h_{i}\right)=\sum_{m=-\infty}^{\infty} c_{m}\left(h_{i}\right) c_{n-m}\left(g_{i}\right)
$$

where the series on the right hand side is convergent since $h_{i}(x)$ is of bounded variation. For convenience we put

$$
c_{n}\left(g_{i} \cdot h_{i}\right)=c_{n}, \quad c_{n}\left(h_{i}\right)=b_{n}, \quad c_{n}\left(g_{i}\right)=a_{n} .
$$

Then from Lemma 1 and (11) we have to consider

$$
\begin{gathered}
\sum_{n=0}^{\infty} \frac{1}{A_{n}^{\alpha+1}}\left|\sum_{k=0}^{n} k A_{n-k}^{\alpha-1}\left(c_{k} e^{i k x}+c_{-k} e^{-i k x}\right)\right| \\
=\sum_{n=0}^{\infty} \frac{1}{A_{n}^{\alpha+1}}\left|\sum_{k=-n}^{n}\right| k\left|A_{n-|k|}^{\alpha-1} c_{k} e^{i k x}\right|
\end{gathered}
$$

(13)

Now let us put

$$
\begin{aligned}
& =\sum_{n=0}^{\infty} \frac{1}{A_{n}^{\alpha+1}}\left|\sum_{k=-n}^{n}\right| k\left|A_{n-|k|}^{\alpha-1} \sum_{m=-\infty}^{\infty} b_{m} a_{k-m} e^{i k x}\right| \\
& \leqq \sum_{m=-\infty}^{\infty}\left|b_{m}\right| \sum_{n=0}^{\infty} \frac{1}{A_{n}^{\alpha+1}}\left|\sum_{k=-n}^{n}\right| k\left|A_{n-|k|}^{\alpha-1} a_{k-m} e^{i k x}\right| .
\end{aligned}
$$

If $m>0$, we have

$$
\rho(m)=\sum_{n=0}^{\infty} \frac{1}{A_{n}^{\alpha+1}}\left|\sum_{k=-n}^{n}\right| k\left|A_{n-|k|}^{\alpha-1} a_{k-m} e^{i k x}\right| .
$$

$$
\rho(m)=\sum_{n=0}^{2 m} \frac{1}{A_{n}^{\alpha+1}}\left|\sum_{k=-n}^{n}\right| k\left|A_{n-|k|}^{\alpha-1}\right| a_{k-m} e^{i k x}\left|+\sum_{n=2 m+1}^{\infty} \frac{1}{A_{n}^{\alpha+1}}\right| \sum_{k=-n}^{n}|k| A_{n-|k|}^{\alpha-1} a_{k-m} e^{i k x} \mid
$$

(14) $=I_{1}+I_{2}$, say.

Since $a_{m}$, being Fourier coefficients of $g_{i}$, is bounded, we get

$$
\begin{aligned}
I_{1} & =\sum_{n=0}^{2 m} \frac{1}{A_{n}^{\alpha+1}}\left|\sum_{k=1}^{n} k A_{n-k}^{\alpha-1}\left(a_{k-m} e^{i(k-m) x}+a_{-k-m} e^{i(-k-m) x}\right)\right| \\
& \leqq \sum_{n=0}^{2 m} \frac{1}{A_{n}^{\alpha+1}}\left\{\sum_{k=1}^{n} k A_{n-k}^{\alpha-1}\left(\left|a_{k-m}\right|+\left|a_{-k-m}\right|\right)\right\} \\
& =O\left\{\sum_{n=0}^{m} \frac{1}{A_{n}^{\alpha+1}} \sum_{k=1}^{n} k A_{n-k}^{\alpha-1}\right\} \\
& =O\left\{\sum_{n=0}^{2 m} \frac{1}{(n+1)^{\alpha+1}}(n+1) \sum_{k=1}^{n} \frac{1}{(n-k+1)^{1-\alpha}}\right\} \\
& =O\left\{\sum_{n=0}^{2 m} \frac{1}{(n+1)^{\alpha+1}}(n+1)(n+1)^{\alpha}\right\} \\
& =O(m) .
\end{aligned}
$$


On the local property of the absolute summability $|C, \alpha|$ for Fourier series.

Proceeding to the estimation of the sum $I_{2}$, we divide its inner sum into four parts :

$$
\begin{aligned}
& \sum_{k=-n}^{n}|k| A_{n-|k|}^{\alpha-1} a_{k-m} e^{i(k-m) x} \\
= & \sum_{k=-n}^{-n+m}|k| A_{n-|k|}^{\alpha-1} a_{k-m} e^{i(k-m) x}+\sum_{k=-n+m}^{n+m}|k-m| A_{n-|k-m|}^{\alpha-1} a_{k-m} e^{i(k-m) x} \\
+ & \sum_{k=-n+m}^{n+m}|k-m|\left(A_{n-|k|}^{\alpha-1}-A_{n-|k-m|}^{\alpha-1}\right) a_{k-m} e^{i(k-m) x} \\
+ & \sum_{k=-n+m}^{n+m}(|k|-|k-m|) A_{n-|k|}^{\alpha-1} a_{k-m} e^{i(k-m) x} \\
= & J_{1}+J_{2}+J_{3}+J_{4}, \quad \text { say, }
\end{aligned}
$$

where we set

$$
A_{k}^{\alpha-1}=0 \quad \text { if } \quad k=-1,-2, \cdots .
$$

Then we have

$$
\begin{aligned}
I_{2} & =\sum_{n=2 m+1}^{\infty} \frac{1}{A_{n}^{\alpha+1}}\left|J_{1}+J_{2}+J_{3}+J_{4}\right| \\
& \leqq \sum_{n=2 m+1}^{\infty} \frac{1}{A_{n}^{\alpha+1}}\left(\left|J_{1}\right|+\left|J_{2}\right|+\left|J_{3}\right|+\left|J_{4}\right|\right) \\
& =K_{1}+K_{2}+K_{3}+K_{4}, \quad \text { say. }
\end{aligned}
$$

The estimation of $K_{1}$ will go as follows:

$$
\begin{aligned}
K_{1} & =\sum_{n=2 m+1}^{\infty} \frac{1}{A_{n}^{\alpha+1}}\left|\sum_{k=-n}^{n+m}\right| k\left|A_{n-|k|}^{\alpha-1} a_{k-m} e^{i(k-m) x}\right| \\
& \leqq \sum_{n=2 m+1}^{\infty} \frac{1}{A_{n}^{\alpha+1}} \sum_{k=n-m}^{n} k A_{n-k}^{\alpha-1}\left|a_{-k-m}\right| \\
& =O\left\{\sum_{n=2 m+1}^{\infty} \frac{1}{n^{\alpha+1}} \sum_{k=n-m}^{n} k(n-k+1)^{\alpha-1}\left|a_{-k-m}\right|\right\} \\
& =O\left\{\sum_{n=2 m+1}^{\infty} \frac{1}{n^{\alpha+1}} n \sum_{k=0}^{m}(m+1-k)^{\alpha-1}\left|a_{-(k+n)}\right|\right\} \\
& =O\left\{\sum_{k=0}^{m}(m+1-k)^{\alpha-1} \sum_{n=2 m+1}^{\infty} \frac{\left|a_{-(k+n)}\right|}{n^{\alpha}}\right\} \\
& =O\left\{\sum_{k=0}^{m}(m+1-k)^{\alpha-1} \sum_{n=2 m+1}^{\infty} \frac{\mid a_{-}(k+n)}{(k+n)^{\alpha}}\left(\frac{k+n}{n}\right)^{\alpha}\right\} .
\end{aligned}
$$

Since $k \leqq 2 m<n,\left(\frac{k+n}{n}\right)^{\alpha}$ does not exceed a constant not depending on $k, m$ and $n$. Therefore 


$$
\begin{aligned}
K_{1} & =O\left\{\sum_{k=0}^{m}(m+1-k)^{\alpha-1} \sum_{n=2 m+k+1}^{\infty} \frac{\left|a_{-n}\right|}{n^{\alpha}}\right\} \\
& =O\left\{\sum_{k=0}^{m}(m+1-k)^{\alpha-1} \sum_{n=1}^{\infty} \frac{\left|a_{-n}\right|}{n^{\alpha}}\right\} \\
& =O\left\{m_{t}^{\alpha} \sum_{n=1}^{\infty} \frac{\left|a_{-n}\right|}{n^{\alpha} \mid}\right\} .
\end{aligned}
$$

The absolute summability $|C, \alpha|$ for Fourier series of $g_{i}(x)$ gives

$$
\sum_{n=1}^{\infty} \frac{\left|a_{-n}\right|}{n^{\alpha}}<\infty
$$

by (8). Hence we get

$$
K_{1}=O\left(m^{\alpha}\right) .
$$

The sum $K_{2}$ will be easily estimated by using the assumption of $g_{i}(x)$ and Lemma 1:

$$
\begin{aligned}
K_{2} & =\sum_{n=2 m+1}^{\infty} \frac{1}{A_{n}^{\alpha+1}}\left|\sum_{k=-n+m}^{n+m}\right| k-m\left|A_{n-|k-m|}^{\alpha-1} a_{k-m} e^{i(k-m) x}\right| \\
& =\sum_{n=2 m+1}^{\infty} \frac{1}{A_{n}^{\alpha+1}}\left|\sum_{k=-n}^{n}\right| k\left|A_{n-|k|}^{\alpha-1} a_{k} e^{i k x}\right| \\
& =O(1) .
\end{aligned}
$$

To estimate the sum $K_{3}$, its inner sum $J_{3}$ will be further divided into two parts :

$$
\begin{aligned}
J_{3} & =\sum_{k=-n+m}^{n+m}|k-m|\left(A_{n-|k|}^{\alpha-1}-A_{n-|k-m|}^{\alpha-1}\right) a_{k-m} e^{i(k-m) x} \\
& =\sum_{k=0}^{2 n}|k-n|\left(A_{n-|k-n+m|}^{\alpha-1}-A_{n-|k-n|}^{\alpha-1}\right) a_{k-n} e^{i(k-n) x} \\
& =\sum_{k=0}^{n}+\sum_{k=n+1}^{2 n}=L_{1}+L_{2}, \quad \text { say. }
\end{aligned}
$$

Then we have

$$
K_{3} \leqq \sum_{n=z m+1}^{\infty} \frac{1}{A_{n}^{\alpha+1}}\left|L_{1}\right|+\sum_{n=2 m+1}^{\infty} \frac{1}{A_{n}^{\alpha+1}}\left|L_{2}\right| .
$$

We shall first estimate the first sum on the right hand side of this inequality :

$$
\begin{gathered}
\sum_{n=2 m+1}^{\infty} \frac{1}{A_{n}^{\alpha+1}}\left|L_{1}\right| \leqq \sum_{n=2 m+1}^{\infty} \frac{1}{A_{n}^{\alpha+1}} \sum_{k=0}^{n}|k-n|\left|A_{n-|k-n+m|}^{\alpha-1}-A_{n-|k-n|}^{\alpha-1}\right|\left|a_{k-n}\right| \\
=\sum_{n=2 m+1}^{\infty} \frac{1}{A_{n}^{\alpha+1}} \sum_{k=0}^{n-m}(n-k)\left|A_{k+m}^{\alpha-1}-A_{k}^{\alpha-1}\right|\left|a_{k-n}\right|+
\end{gathered}
$$


Since

$$
\begin{aligned}
& +\sum_{n=2 m+1}^{\infty} \frac{1}{A_{n}^{\alpha+1}} \sum_{k=n-m+1}^{n}(n-k)\left|A_{2 n-m-k}^{\alpha-1}-A_{k}^{\alpha-1}\right|\left|a_{k-n}\right| \\
& =M_{1}+M_{2}, \quad \text { say. }
\end{aligned}
$$

$$
\left|A_{k+m}^{\alpha-1}-A_{k}^{\alpha-1}\right|=\left|\sum_{j=0}^{m-1} A_{k+1+j}^{\alpha-2}\right|=O\left(\frac{m}{(k+1)^{2-\alpha}}\right),
$$

we have

$$
\begin{aligned}
M_{1} & =O\left\{\sum_{n=2 m+1}^{\infty} \frac{1}{n^{\alpha+1}} \sum_{k=0}^{n-m}(n-k) \frac{m}{(k+1)^{2-\alpha}}\left|a_{k-n}\right|\right\} \\
& =O\left\{m \sum_{k=0}^{\infty} \frac{1}{(k+1)^{2-\alpha}} \sum_{n=k+m}^{\infty} \frac{(n-k)}{n^{\alpha+1}}\left|a_{k-n}\right|\right\} \\
& =O\left\{m \sum_{k=0}^{\infty} \frac{1}{(k+1)^{2-\alpha}} \sum_{n=0}^{\infty} \frac{(m+n)^{\alpha+1}}{(k+m+n)^{\alpha+1}(m+n)^{\alpha}}\right\} \\
& =O\left\{m \sum_{k=0}^{\infty} \frac{1}{(k+1)^{2-\alpha}} \sum_{n=1}^{\infty} \frac{\left|a_{-n}\right|}{n^{\alpha}}\right\} \\
& =O\left\{m \sum_{n=1}^{\infty} \frac{\left|a_{-n}\right|}{n^{\alpha}}\right\} .
\end{aligned}
$$

For the sum $M_{2}$, we have

$$
\begin{aligned}
M_{2} & =\sum_{n=2 m+1}^{\infty} \frac{1}{A_{n}^{\alpha+1}} \sum_{k=n-m+1}^{n}(n-k)\left|A_{2 n-m-k}^{\alpha-1}-A_{k}^{\alpha-1}\right|\left|a_{k-n}\right| \\
& =\sum_{n=2 m+1}^{\infty} \frac{1}{A_{n}^{\alpha+1}} \sum_{k=1}^{m}(m-k)\left|A_{n-k}^{\alpha-1}-A_{n-m+k}^{\alpha-1}\right|\left|a_{-m+k}\right| \\
& =O\left\{\sum_{n=2 m+1}^{\infty} \frac{1}{n^{\alpha+1}} \sum_{k=1}^{m}(m-k) \frac{1}{(n-m+k)^{1-\alpha}}\left|a_{-m+k}\right|\right\} \\
& =O\left\{\sum_{n=2 m+1}^{\infty} \frac{1}{n^{\alpha+1}} \sum_{k=1}^{m} \frac{(m-k+1)^{\alpha+1}\left|a_{-m+k}\right|}{(n-m+k)^{1-\alpha}(m-k+1)^{\alpha}}\right\} \\
& =O\left\{m^{\alpha+1} \sum_{n=2 m+1}^{\infty} \frac{1}{n^{\alpha+1}(n-m)^{1-\alpha}} \sum_{k=1}^{\infty} \frac{\left|a_{-k}\right|}{k^{\alpha}}\right\} \\
& =O\left\{m^{\alpha+1} \sum_{n=2 m+1}^{\infty} \frac{1}{(n-m)^{2}} \sum_{k=1}^{\infty} \frac{\left|a_{-k}\right|}{k^{\alpha}}\right\} \\
& =O\left\{m^{\alpha} \sum_{n=1}^{\infty} \frac{\left|a_{-k}\right|}{k^{\alpha}}\right\} .
\end{aligned}
$$

By the assumption of $g_{i}(x)$ and (8) we have

$$
\begin{aligned}
& M_{1}=O(m), \\
& M_{2}=O\left(m^{\alpha}\right)=O(m) .
\end{aligned}
$$

Hence we get

$$
\sum_{n=2 m+1}^{\infty} \frac{1}{A_{n}^{\alpha+1}}\left|L_{1}\right|=O(m) .
$$


Similarly

$$
\sum_{n=2 m+1}^{\infty} \frac{1}{A_{n}^{\alpha+1}}\left|L_{2}\right|=O(m) .
$$

Thus (20), (22) and (23) give

$$
K_{3}=O(m) .
$$

Finally, let us estimate the sum $K_{4}$. Taking into account that

$$
\begin{aligned}
\left|J_{4}\right| & =\left|\sum_{k=-n+m}^{n+m}(|k|-|k-m|) A_{n-|k|}^{\alpha-1} a_{k-m} e^{i(k-m) x}\right| \\
& \leqq m \sum_{k=-n+m}^{n+m} A_{n-|k|}^{\alpha-1}\left|a_{k-m}\right| \\
& =m \sum_{k=-n}^{n} A_{n-|m+k|}^{\alpha-1}\left|a_{k}\right| \\
& =m \sum_{k=0}^{n-m} A_{n-(m+k)}^{\alpha-1}\left|a_{k}\right|+m \sum_{k=1}^{n} A_{n-|m-k|}^{\alpha-1}\left|a_{-k}\right| \\
& =N_{1}+N_{2}, \quad \text { say, }
\end{aligned}
$$

we obtain

$$
K_{4} \leqq \sum_{n=2 m+1}^{\infty} \frac{1}{A_{n}^{\alpha+1}}\left|N_{1}\right|+\sum_{n=2 m+1}^{\infty} \frac{1}{A_{n}^{\alpha+1}}\left|N_{2}\right| .
$$

For the first sum on the right hand side of (25) we have

$$
\begin{aligned}
\sum_{n=2 m+1}^{\infty} \frac{1}{A_{n}^{\alpha+1}}\left|N_{1}\right| & =O\left\{m \sum_{n=2 m+1}^{\infty} \frac{1}{(n+1)^{\alpha+1}} \sum_{k=0}^{n-m}(n-m-k+1)^{\alpha-1}\left|a_{k}\right|\right\} \\
& =O\left\{m \sum_{k=0}^{\infty}\left|a_{k}\right| \sum_{n=m+k}^{\infty} \frac{1}{(n+1)^{\alpha+1}}\right\} \\
& =O\left\{m \sum_{k=0}^{\infty} \frac{\left|a_{k}\right|}{(m+k)^{\alpha}}\right\} \\
& =O\left\{m \sum_{k=1}^{\infty} \frac{\left|a_{k}\right|}{k^{\alpha}}\right\} .
\end{aligned}
$$

Similarly

$$
\sum_{n=2 m+1}^{\infty} \frac{1}{A_{n}^{\alpha+1}}\left|N_{2}\right|=O\left\{m \sum_{k=1}^{\infty} \frac{\left|a_{k}\right|}{k^{\alpha}}\right\}
$$

But by the assumption the series $\sum_{k=1}^{\infty} \frac{\left|a_{k}\right|}{k^{\alpha}}$ converges. Hence from (25), (26) and (27) we get

$$
K_{4}=O(m) .
$$

Combining the above estimations (17), (18), (19), (24) and, (28) we obtain

$$
I_{2}=O(m) \text {. }
$$


Therefore, if $m>0$, we have

$$
\rho(m)=O(m)
$$

by (14), (15) and (29),

A similar result for $m \leqq 0$ can be proved in exactly the same manner, and therefore by (10)

$$
\begin{aligned}
\sum_{m=-\infty}^{\infty}\left|b_{m}\right| \rho(m) & =O\left\{\sum_{m=-\infty}^{\infty}|m|^{-3}|m|\right\} \\
& =O\left\{\sum_{m=-\infty}^{\infty}|m|^{-2}\right\}<\infty,
\end{aligned}
$$

so that by (13)

$$
\sum_{n=0}^{\infty} \frac{1}{A_{n}^{\alpha+1}}\left|\sum_{k=0}^{n} k A_{n-k}^{\alpha-1}\left(c_{k} e^{i k x}+c_{-k} e^{-i k x}\right)\right|<\infty .
$$

From this and Lemma 1 it follows that the Fourier series of $h_{i}(x) g_{i}(x)$ is absolutely summable $|C, \alpha|$, and so by (11) $f(x)$, being the sum of a finite number of functions having Fourier series which are absolutely summable $|C, \alpha|$, must also have a Fourier series which is absolutely summable $|C, \alpha|$, where $0<\alpha<1$.

This completes the proof of our theorem.

\section{Mathematical Institute, Tokyo Metropolitan University.}

\section{References}

[1] L.S. Bosanquet, The absolute Cesàro summability of Fourier series, Proc. London Math. Soc., 11 (1936), pp. 517-528.

[2] L.S. Bosanquet and H. Kestelman, The absolute convergence of series of integrals, Proc. London Math. Soc., (2) 45 (1939), pp. 88-97.

[3] M.E. Kogbetliantz, Sur les séries absolument sommables par la méthode des moyennes arithmétiques, Bull. Sci. Math., (2) 49 (1925), pp. 234-256.

[4] V.A. Magarik, On the summability $|C, \alpha|$ of Fourier series, Moskv. Gos. Univ. Uc. Zap. 181, Mat., 8 (1956), pp. 183-196. (in Russian).

[5] W.C. Randels, On the absolute summability of Fourier Series, Duke Math. J., 7 (1940), pp. 204-207.

[6] A. Zygmund, Trigonometrical series, Warszawa, (1935). 